\title{
The Predictive Value of Precise-Dapt Score for Mortality in Patients with Acute Decompansed Heart Failure
}

\author{
Mevlut Demir' ${ }^{1}$ Ahmet Korkmaz², Bekir Demirtas ${ }^{3}$ \\ 'Department of Cardiology, Kutahya Health Science University, Kutahya, ${ }^{2}$ Department of Cardiology, Ankara City Hospital, Ankara, ${ }^{3}$ Department of Cardiology, \\ Cankiri State Hospital, Cankiri, Turkey \\ ORCID: \\ Mevlut Demir: 0000-0002-7484-9969 \\ Ahmet Korkmaz: 0000-0003-2672-5109 \\ Bekir Demirtas: 0000-0002-6266-2291
}

\section{Abstract}

Introduction: Acute decompensated heart failure (ADHF) is an emergency clinical syndrome defined as a sudden worsening of heart failure. PREdicting bleeding Complications In patients undergoing Stent implantation and subsEquent Dual Anti-Platelet Therapy (PRECISE-DAPT) score is a new scoring used in the management of duration of dual-antiplatelet therapy after coronary intervention. We presented the hypothesis that this scoring can be used as a predictor of mortality in heart failure. Objective: In this study, the correlation between mortality and PRECISE-DAPT score will be analyzed in patients diagnosed with ADHF. Materials and Methods: A total of 114 patients hospitalized with a diagnosis of ADHF were included in this study. The patients were divided into two groups: PRECISE-DAPT score $\geq 25$ and PRECISE-DAPT score $<25$, and these groups were evaluated in terms of correlation with early ( $<6$ months), late ( $>6$ months) and overall mortality. Results: According to univariate analysis, it was found that PRECISE-DAPT score was significantly related with early $(P<0.001)$, late $(P<0.001)$, and overall $(P<0.001)$ mortality. Multivariate Cox regression analysis showed that PRECISE-DAPT was independently associated with late (hazard ratio: 6.6; $95 \%$ confidence interval $[\mathrm{CI}] 1.6-27.3 ; P=0.009$ ) and overall (hazard ratio: $11.3 ; 95 \%$ CI $3.2-40.9 ; P<0.001$ ) mortality. According to KaplanMeier curve, those with a score of $\geq 25$ were shown to have significantly higher mortality. The predictive ability for the PRECISE-DAPT score threshold value of 25 was investigated in 3 mortality subgroups. Conclusion: The PRECISE-DAPT score may be a significant independent predictor of mortality in patients with ADHF. Limitations: This study is subject to the limitations inherent to a retrospective study and the sample size in our study is relatively small.

Keywords: Heart failure, mortality, score

\section{INTRODUCTION}

Acute decompensated heart failure (ADHF) is an emergency clinical syndrome defined as a sudden worsening of heart failure symptoms (dyspnea, orthopnea, swelling of the lower extremity). ADHF, which accounts for most of the sudden-onset dyspnea cases in the elderly patient population, constitutes about $40 \%$ of the elderly patients admitting to the emergency department with this complaint in the USA. ${ }^{[1,2]}$ While about $20 \%$ of these patients have no history of previous cardiac disease, the remaining vast majority have preexisting systolic or diastolic heart failure with or without valvular

Received: 18-03-2021 Revised: 01-06-2021 Accepted: 15-06-2021

Published Online: 25-09-2021

\begin{tabular}{|l|l|}
\hline \multicolumn{3}{|c|}{ Access this article online } \\
\hline Quick Response Code: & Website: \\
& \\
http://www.ijcva.com
\end{tabular}

regurgitation or coronary artery disease (CAD) ${ }^{[3]}$ Despite many studies and treatment efforts to reduce mortality rates in heart failure with an annual treatment cost of about $\$ 40$ billion, decompensated heart failure has annual mortality rates ranging from $25 \%$ to $40 \%$ in the population aged 65 years or over. ${ }^{[4,5]}$

The PREdicting bleeding Complications In patients undergoing Stent implantation and subsEquent Dual Anti-Platelet Therapy (PRECISE-DAPT) score is a new scoring used

Address for correspondence: Dr. Mevlut Demir Department of Cardiology, Kutahya Health Science University, 43020 Kutahya, Turkey. E-mail: drmevlutdemir@hotmail.com

This is an open access journal, and articles are distributed under the terms of the Creative Commons Attribution-NonCommercial-ShareAlike 4.0 License, which allows others to remix, tweak, and build upon the work non-commercially, as long as appropriate credit is given and the new creations are licensed under the identical terms.

For reprints contact: WKHLRPMedknow_reprints@wolterskluwer.com

How to cite this article: Demir M, Korkmaz A, Demirtas B. The predictive value of precise-dapt score for mortality in patients with acute decompansed heart failure. Int J Cardiovasc Acad 2021;7:70-7. 
in the management of duration of dual-antiplatelet therapy after coronary intervention, consisting of age, hemoglobin, creatinine clearance, white blood cell, and history of previous spontaneous bleeding factors. ${ }^{[6]}$

We presented the hypothesis that this scoring can be used as a predictor of mortality in heart failure as these parameters used in the scoring are also contributing factors in the prognosis of heart failure. To the best of our knowledge, there is no data in the literature on the significance of PRECISE-DAPT for ADHF patients. In this study, the correlation between mortality and PRECISE-DAPT score will be analyzed in patients diagnosed with ADHF. This article was previously presented as a meeting abstract at the TSC Clinical Studies Application and Education Project Group-(STATISTANBUL 2020) February 29, 2020.

\section{Materials and Methods}

\section{Patients}

In this study, patients who presented to the emergency department with acute decompensated cardiac failure findings and were admitted to the cardiology clinic with this diagnosis between July 2012 and February 2014 were retrospectively scanned through the records, emergency and cardiology department files, and electronic information system. Patients who could not be diagnosed definitively because of their insignificant symptoms were excluded from the study. It was found that 114 of these patients met the study inclusion criteria.

\section{Inclusion and exclusion criteria}

Patients evaluated in the emergency department with the complaints of newly developed or rapidly worsening shortness of breath, palpitations, orthopnea, swelling in the feet and found to have diffuse crepitant rales in bilateral lower or middle zones and pretibial edema on physical examination and congestion and pleural fluid findings on chest X-ray and responded to diuretic therapy and admitted to the cardiology clinic with the diagnosis of ADHF, according to the Framingham Heart Failure Diagnostic Criteria ${ }^{[7]}$ were included in the study by reviewing emergency room examination form and the cardiology admission file. Those with chronic lung disease, congenital heart disease, severe valvular dysfunction, cardiac tamponade, cardiac thrombus and vegetation, kidney failure (glomerular filtration rate $[\mathrm{GFR}]<30$ ), renal artery stenosis and pheochromocytoma, severe liver failure, those aged under 18 years, patients with malignant neoplasm and infectious and inflammatory disease were excluded from the study. Patients who had uncertain complaints and did not receive a definitive diagnosis were not included in the study. In addition, patients with coronary stenosis over $60 \%$ on coronary angiography performed on suspicion of coronary ischemia or ischemia on myocardial perfusion scintigraphy (MPS) were excluded from the study.

\section{Data analysis}

All patients underwent electrocardiography, routine hemogram and biochemistry analysis, arterial blood gas analysis, electrocardiography, chest X-ray, and transthoracic echocardiography during the admission. The duration of complaints, number of hospitalizations, treatment compliance, history of chronic disease, medical treatment used, and history of smoking or substance use of the patients were noted.

The patients were evaluated for the first time in the emergency department with Philips portable echocardiography and for the second time after admission to the cardiology ward with GE Vivid 3 Pro echocardiography. First parasternal, then apical four chambers and two-chamber along with valvular functions, systolic and diastolic functions, and left and right ventricles were evaluated in the patients placed in the left lateral and supine positions. The patients with orthopnea underwent echocardiographic evaluation with the bed elevated to $45^{\circ}$. In addition, left ventricular ejection fractions were calculated using the modified Simpson technique.

During the admission of the patients, routine urea, creatinine, GFR, alanine aminotransferase, aspartate aminotransferase, total cholesterol, high-density lipoprotein, low-density lipoprotein, total protein, thyroid-stimulating hormone, troponin, admission blood glucose levels were studied in the biochemistry laboratory. Moreover, peripheral venous blood samples were studied in the hematology laboratory and complete blood cell parameters were calculated using an automated complete blood count device (BeckmanCoulter, Brea, CA, USA). The patients with acute coronary ischemia and troponin elevation were primarily evaluated for coronary ischemia by coronary angiography or MPS, and those found to have coronary ischemia were excluded from the study. The data to be evaluated were obtained from the patient files via the electronic information recording system of our hospital. The follow-up parameters included outpatient clinic follow-up examinations and phone calls. All data were arranged within the framework of the case registration form.

The PRECISE-DAPT scores of the patients were calculated using a web calculator (http://www.precisedaptscore.com). The patients were divided into two groups: PRECISE-DAPT score $\geq 25$ and PRECISE-DAPT score $<25$, and these groups were evaluated in terms of correlation with mortality. The diastolic blood pressures of the patients were categorized as Grade 1 hypertension: 90-99 mmhg, Grade 2 hypertension: 100-109 mmhg, Grade 3 hypertension: $\geq 110 \mathrm{mmhg}$. The reference range value for troponin was taken as $0-0.034 \mathrm{ng} / \mathrm{ml}$.

\section{Statistical analysis}

We analyzed the data with SPSS, version 21.0 for Windows (IBM Corp., Armonk, NY). Numerical variables were summarized as mean \pm standard deviation, median (minimum-maximum) values. Categorical variables were shown with numbers and percentages. The numerical variables analyzed in the study were described with mean and standard deviation, and while categorical variables were described with frequency and percentage values. The correlation between categorical variables was analyzed with the Chi-square test. The difference between the two independent means was analyzed 
with the Mann-Whitney U Test. Two independent means were compared with the Mann-Whitney U Test. In the analysis of survival, Kaplan-Meier Analysis and multivariate Cox Regression Analysis methods were used. The study was conducted at a confidence level of $95 \%$. The level of significance was set at $P<0.05$.

\section{Ethical statement}

The study protocol was approved by the Local Ethics Committee. Patient Consent Declaration was obtained from the patients. The study was conducted in accordance with the Helsinki Declaration.

\section{RESULTS}

Our study included patients who were hospitalized with the diagnosis of ADHF, Class IV according to New York Heart Association Functional Classification for Heart Failure, ( $n=114$, mean age $67.4 \pm 10$ ) by evaluating retrospectively.

The patients were divided into two groups based on their PRECISE-DAPT scores (PRECISE-DAPT $\geq 25$ vs. PRECISE-DAPT <25). Of the patients, 63 (55\%) had a PRECISE-DAPT score of $\geq 25$ and 51 (45\%) had a PRECISE-DAPT score of $<25$. The median (interquartile range) follow-up time of the patients was 19 (25) months. The demographic and clinical characteristics of the patients are given in Table 1.

The mean value of ejection fraction of patients is $29.7 \% \pm 6.3 \%$ and $80.7 \%$ of these consist of ischemic heart disease. Furthermore, it can be said that acute heart failure develops secondary to hypertension due to very high systolic and diastolic pressures of patients.

There was no significant correlation between the patients with a PRECISE-DAPT score $\geq 25$ and PRECISE-DAPT score $<25$ in terms of gender, diabetes mellitus, hypertension, and cerebrovascular event frequency. It was found that the ages of the patients with a PRECISE-DAPT score of $\geq 25$ were significantly higher than that of PRECISE-DAPT $<25$ group $(61.3 \pm 10$ vs. $72.4 \pm 7.9 ; P<0.01)$. Moreover, it was found that ischemic cardiomyopathy $(95.2 \%$ vs. $4.8 \% ; P<0.01)$ and bleeding history (74.6\% vs. $25.4 \%$ $P<0.01)$ were significantly more frequent in those with a PRECISE-DAPT score of $\geq 25$ had, and that their diastolic

\begin{tabular}{|c|c|c|c|c|}
\hline & Toplam $(n=114)$ & Precise-DAPT $<25(n=51)$ & Precise-DAPT $\geq 25(n=63)$ & $P$ \\
\hline Age, years $($ mean \pm SD) & $67.4 \pm 10.4$ & $61.3 \pm 10.0$ & $72.4 \pm 7.9$ & $<0.001$ \\
\hline \multicolumn{5}{|l|}{ Sex, $n(\%)$} \\
\hline Female & $51(44.7)$ & $21(41.2)$ & $30(47.6)$ & 0.492 \\
\hline Male & $63(55.3)$ & $30(58.8)$ & $33(52.4)$ & \\
\hline Prior bleeding, $n(\%)$ & $16(14)$ & 0() & $16(25.4)$ & $<0.001$ \\
\hline In-hospital mortality, $n(\%)$ & $10(8.8)$ & 0() & $10(15.9)$ & 0.002 \\
\hline Duration of follow-up, median (IQR) & $19(25)$ & $25(21)$ & $9(20.5)$ & $<0.001$ \\
\hline \multicolumn{5}{|l|}{ Mortality, $n(\%)$} \\
\hline Survivors & $64(56.1)$ & $46(90.2)$ & $18(28.6)$ & $<0.001$ \\
\hline Early mortality ( $\leq 6$ months) & $26(22.8)$ & $1(2)$ & $25(39.7)$ & \\
\hline Late mortality ( $>6$ months) & $24(21.1)$ & $4(7.8)$ & $20(31.7)$ & \\
\hline \multicolumn{5}{|l|}{ Total population, $n(\%)$} \\
\hline Survivors & $65(57)$ & $47(92.2)$ & $18(28.6)$ & $<0.001$ \\
\hline Non-survivors & $49(43)$ & $4(7.8)$ & $45(71.4)$ & \\
\hline Hypertension & $87(76.3)$ & $37(72.5)$ & $50(79.4)$ & 0.395 \\
\hline Diabetes mellitus & $46(40.4)$ & $17(33.3)$ & $29(46)$ & 0.169 \\
\hline Hyperlipidemia & $49(43)$ & $23(45.1)$ & $25(39.7)$ & 0.681 \\
\hline Prior coronary artery disease & $73(64)$ & $31(60.8)$ & $42(66.7)$ & 0.515 \\
\hline Prior cerebrovascular event & $10(8.8)$ & $4(7.8)$ & $6(9.5)$ & $>0.999$ \\
\hline \multicolumn{5}{|l|}{ Type of cardiomyopathy, $n(\%)$} \\
\hline Ischemic & $92(80.7)$ & $32(62.7)$ & $60(95.2)$ & $<0.001$ \\
\hline Nonischemic & $22(19.3)$ & $19(37.3)$ & $3(4.8)$ & \\
\hline Sistolic blood pressure $(\mathrm{mmhg})($ mean $\pm \mathrm{SD})$ & $213.3 \pm 24.4$ & $210.1 \pm 24.9$ & $215.9 \pm 23.9$ & 0.161 \\
\hline Diastolic blood pressure (mmhg) (mean \pm SD) & $111.1 \pm 11.4$ & $108.9 \pm 10.8$ & $112.8 \pm 11.6$ & 0.033 \\
\hline Heart rate (beats per min) & $115.7 \pm 10.2$ & $117.5 \pm 11.7$ & $114.2 \pm 8.5$ & 0.315 \\
\hline Troponin I (ng/ml) (mean \pm SD) & $0.35 \pm 0.65$ & $0.28 \pm 0.63$ & $0.40 \pm 0.66$ & 0.013 \\
\hline Blood urea nitrogen (mg/dl) & $24.3 \pm 10.5$ & $20.7 \pm 8.8$ & $27.3 \pm 10.9$ & 0.073 \\
\hline Ejection fraction $\%(\operatorname{mean} \pm \mathrm{SD})$ & $29.7 \pm 6.3$ & $29.3 \pm 6.3$ & $30.1 \pm 6.2$ & 0.515 \\
\hline Precise-DAPT score (mean \pm SD) & $33.4 \pm 50.8$ & $18.1 \pm 5.4$ & $45.9 \pm 65.8$ & $<0.001$ \\
\hline GWTG-heart failure risk score & $33.2 \pm 5.5$ & $29.6 \pm 4.3$ & $36.1 \pm 4.5$ & 0.061 \\
\hline
\end{tabular}

DAPT: Dual antiplatelet therapy, SD: Standard deviation, IQR: Interquartile range, GWTG: Get with the guidelines 
blood pressures $(108.9 \pm 10$ vs. $112.8 \pm 11.6 ; P=0.033)$ were significantly higher at the time of admission. The patients with a PRECISE-DAPT of $\geq 25$ were found to have significantly higher troponin values $(0.28 \pm 0.63 \mathrm{vs} .0 .40 \pm 0.66$; $P=0.013$ ) (reference troponin value: $0-0.034 \mathrm{ng} / \mathrm{ml}$ ) at the time of admission [Table 1].

The mortality of the patients was analyzed by categorizing into 3 groups as early ( $<6$ months), late ( $>6$ months), and total. When the patients with early mortality were evaluated, it was found that PRECISE-DAPT score, bleeding history, diastolic blood pressure, admission troponin value, ischemic CMP frequency were factors affecting early mortality. However, when the factors affecting early mortality were evaluated by multivariate analysis, it was found that these values were not an independent risk factor [Table 2].

When the patients with late mortality ( $>6$ months) were evaluated, it was found that PRECISE-DAPT score, age, bleeding history, admission diastolic blood pressure, and admission troponin values were factors affecting late mortality. When these factors were evaluated by multivariate analysis, it was found that PRECISE-DAPT score was an independent risk factor in the patients with late mortality, and that mortality was 6.6 times higher in the patients with a PRECISE-DAPT score of $\geq 25 \%$ [Table 3].

When the total mortality of the patients was evaluated, it was found that the patients with a PRECISE-DAPT score of $\geq 25$ had significantly higher mortality [Table 2]. It was also found that the patients who died had more frequent bleeding history, higher diastolic blood pressures, and higher troponin values.
When the patients were evaluated with multivariate analysis in terms of total mortality, the patients with a PRECISE-DAPT score of $\geq 25$ were shown to have 11.3 times (3.2-40.9; $P<0.01)$ higher mortality rate and their bleeding rates were independently significantly higher [Table 4].

When early, late, and total mortality values and PRECISE-DAPT were evaluated with the Kaplan-Meier curve, those with a score of $\geq 25$ were shown to have significantly higher mortality [Figures 1-3].

The predictive ability for the PRECISE-DAPT score threshold value of 25 was investigated in all patients and early and late mortality subgroups. The area under the curve values obtained from receiver operating characteristic Analysis were calculated. Accordingly, it was found to have a high predictive power for all three groups [Figure 4].

In cases where the PRECISE-DAPT value is calculated as 25 and higher, the predictive validity for mortality was calculated when the survived patients and all died patients were included (91.8\%), when the survived patients and the patients died before 6 months (96.2\%) were included, and when the survived patients and the patients died after 6 months were included (83.3\%) [Table 5].

In cases, where the PRECISE-DAPT value is calculated below 25 , the predictive validity for mortality was calculated when the survived patients and all died patients were included $(72.3 \%)$, when the survived patients and the patients died before 6 months were included (71.9\%), and when the survived patients and the patients died after 6 months were included (71.9\%) [Table 5].

Table 2: Significant predictors of early mortality in univariable and multivariable cox regression analyses

\begin{tabular}{|c|c|c|c|c|c|c|}
\hline & \multicolumn{3}{|c|}{ Univariate } & \multicolumn{3}{|c|}{ Multivariate } \\
\hline & Median (months) & $95 \% \mathrm{Cl}$ & $P$ (Log-rank) & HR & $95 \% \mathrm{Cl}$ & $P$ \\
\hline Age (years) $\geq 69.5$ & - & - & 0.001 & 1.0 & $0.4-2.7$ & 0.962 \\
\hline Prior bleeding $(+)$ & 1.5 & $0.4-2.6$ & $<0.001$ & 2.4 & $1.0-5.8$ & 0.058 \\
\hline Ischemic CMP (+) & - & - & 0.004 & 16476 & $0-2.5 \mathrm{E}+87$ & 0.921 \\
\hline Diastolic blood pressure $(\mathrm{mmhg}) \geq 110$ & - & - & 0.065 & 1.5 & $0.6-4.1$ & 0.415 \\
\hline $\operatorname{Tn} \mathrm{I}(\mathrm{ng} / \mathrm{ml}) \geq 0.045$ & 63.0 & - & 0.007 & 1.9 & $0.8-4.4$ & 0.159 \\
\hline Precise DAPT $\geq 25$ & 5.0 & $1.3-8.7$ & $<0.001$ & 210865 & $0-2.5 E+95$ & 0.908 \\
\hline Overall & 63.0 & - & & & & \\
\hline
\end{tabular}

Tn: Troponin, CMP: Cardiomyopathy, CI: Confidence interval, HR: Hazard ratio, DAPT: Dual antiplatelet therapy

Table 3: Significant predictors of late mortality in univariable and multivariable cox regression analyses

\begin{tabular}{|c|c|c|c|c|c|c|}
\hline & \multicolumn{3}{|c|}{ Univariate } & \multicolumn{3}{|c|}{ Multivariate } \\
\hline & Median (months) & $95 \% \mathrm{Cl}$ & $P$ (Log-rank) & HR & $95 \% \mathrm{Cl}$ & $P$ \\
\hline Age (years) $\geq 69.5$ & 53.0 & $18.4-87.6$ & 0.013 & 0.96 & $0.4-2.6$ & 0.959 \\
\hline Prior bleeding $(+)$ & 9.5 & 8.1-10.9 & $<0.001$ & 4.4 & $1.6-11.7$ & 0.003 \\
\hline Ischemic CMP (+) & - & - & 0.054 & 1.2 & $0.3-4.6$ & 0.793 \\
\hline Diastolic blood pressure $(\mathrm{mmhg}) \geq 110$ & 53.0 & $33.9-72.1$ & 0.029 & 2.5 & $0.8-7.5$ & 0.112 \\
\hline $\operatorname{Tn} \mathrm{I}(\mathrm{ng} / \mathrm{ml}) \geq 0.045$ & - & - & 0.037 & 1.5 & $0.6-3.6$ & 0.395 \\
\hline Precise DAPT $\geq 25$ & 22.0 & $2.5-41.5$ & $<0.001$ & 6.6 & $1.6-27.3$ & 0.009 \\
\hline Overall & - & - & & & & \\
\hline
\end{tabular}

Tn: Troponin, CMP: Cardiomyopathy, CI: Confidence interval, HR: Hazard ratio, DAPT: Dual anti-platelet therapy 
Table 4: Significant predictors of overall mortality in univariable and multivariable cox regression analyses

\begin{tabular}{|c|c|c|c|c|c|c|}
\hline & \multicolumn{3}{|c|}{ Univariate } & \multicolumn{3}{|c|}{ Multivariate } \\
\hline & Median (months) & $95 \% \mathrm{Cl}$ & $P$ (Log-rank) & HR & $95 \% \mathrm{Cl}$ & $P$ \\
\hline Age (years) $\geq 69.5$ & 14.5 & $5.1-23.9$ & $<0.001$ & 1.1 & $0.6-2.2$ & 0.716 \\
\hline Prior bleeding $(+)$ & 6.0 & $2.7-9.3$ & $<0.001$ & 2.2 & $1.2-4.2$ & 0.017 \\
\hline Ischemic CMP (+) & 24.0 & $1.8-46.2$ & 0.001 & 1.8 & $0.5-6.4$ & 0.340 \\
\hline Diastolic blood pressure $(\mathrm{mmhg}) \geq 110$ & 24.0 & $3.8-44.2$ & 0.009 & 1.9 & $0.9-4.0$ & 0.093 \\
\hline $\operatorname{Tn} \mathrm{I}(\mathrm{ng} / \mathrm{ml}) \geq 0.045$ & 17.0 & $7.1-26.9$ & 0.003 & 1.6 & $0.9-3.0$ & 0.119 \\
\hline Precise DAPT $\geq 25$ & 10.0 & $4.4-15.6$ & $<0.001$ & 11.3 & $3.2-40.9$ & $<0.001$ \\
\hline Overall & 53.0 & $9.0-96.7$ & & & & \\
\hline
\end{tabular}

Tn: Troponin, CMP: Cardiomyopathy, CI: Confidence interval, HR: Hazard ratio, DAPT: Dual antiplatelet therapy

\section{Table 5: Cut-off characteristics}

\begin{tabular}{lccr}
\hline Precise DAPT $\geq 25$ & Overall & Early & Late \\
\hline Sensitivity $(95 \% \mathrm{CI})$ & $0.918(0.804-0.977)$ & $0.962(0.804-0.999)$ & $0.833(0.626-0.953)$ \\
Specificity $(95 \% \mathrm{CI})$ & $0.723(0.598-0.827)$ & $0.719(0.592-0.824)$ & $0.719(0.592-0.824)$ \\
PPV $(95 \% \mathrm{CI})$ & $0.714(0.626-0.789)$ & $0.581(0.482-0.674)$ & $0.526(0.419-0.631)$ \\
NPV $(95 \% \mathrm{CI})$ & $0.922(0.820-0.968)$ & $0.979(0.870-0.997)$ & $0.920(0.823-0.966)$ \\
Accuracy $(95 \% \mathrm{CI})$ & $0.807(0.723-0.875)$ & $0.789(0.690-0.868)$ & $0.750(0.646-0.836)$ \\
\hline
\end{tabular}

DAPT: Dual antiplatelet therapy, CI: Confidence interval, PPV: Positive predictive value, NPV: Negative predictive value

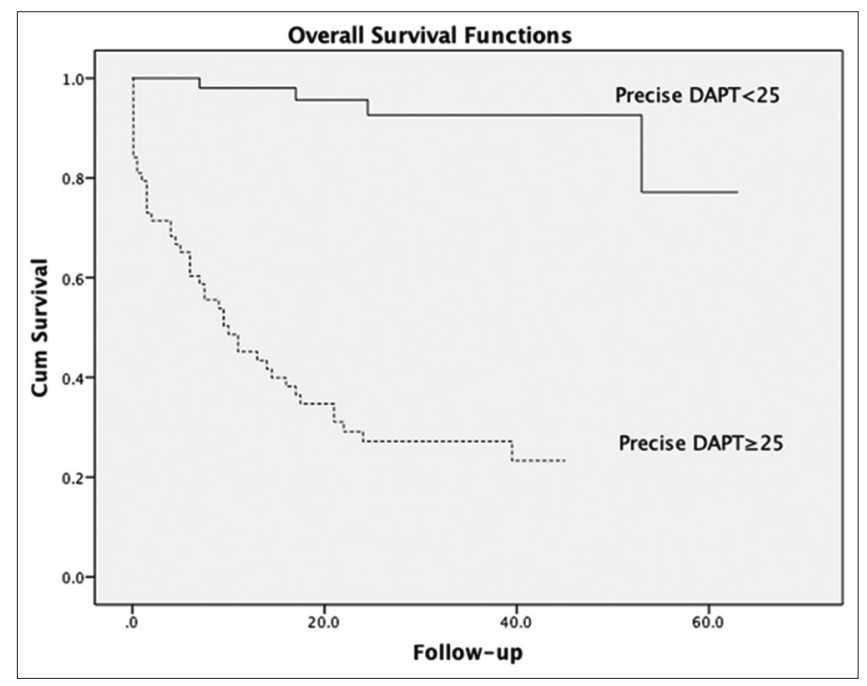

Figure 1: Kaplan Meier overall survival curve according to PREdicting bleeding Complications In patients undergoing Stent implantation and subsEquent Dual Anti-Platelet Therapy

Accordingly, it was found that the threshold value had a high predictive power for all three different cases analyzed in our study.

\section{Discussion}

In this study, we demonstrated that PRECISE-DAPT score is independently correlated with mortality in patients hospitalized with ADHF. As far as we know, our study is the first study evaluating the correlation between PRECISE-DAPT and mortality in patients with acute decompression heart failure.

The incidence of heart failure has increased due to the increasing elderly population and prolonged surveillance

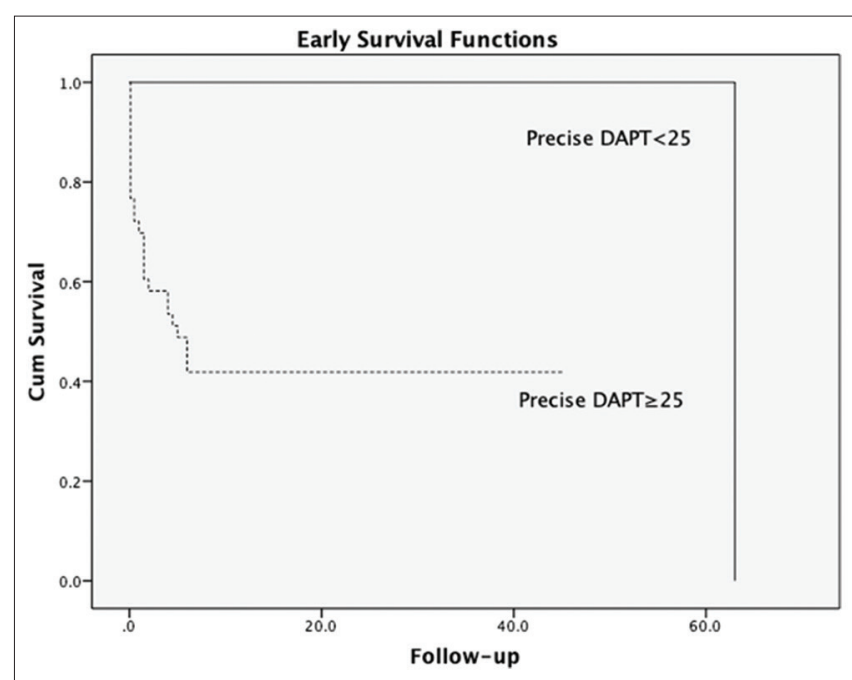

Figure 2: Kaplan Meier early survival curve according to PREdicting bleeding Complications In patients undergoing Stent implantation and subsEquent Dual Anti-Platelet Therapy

of cardiovascular diseases with modern treatment methods. It is considered global health problem. In addition, due to the need for life-long treatment, frequent hospitalization, different device, and medical treatment options brought about high costs on health economics. ${ }^{[8,9]}$ Despite the increase in the functional capacity of the patients and the decrease in the frequency of hospitalization with the treatment protocol recommended by the guidelines, the hospitalization and mortality rates of the disease are higher than the study data due to patient-treatment incompatibility and the lack of adequate treatment. ${ }^{[10]}$ Moreover, due to the multifactorial heterogeneous pathophysiology of heart failure, many factors affecting the short and long-term prognosis have been reported. 


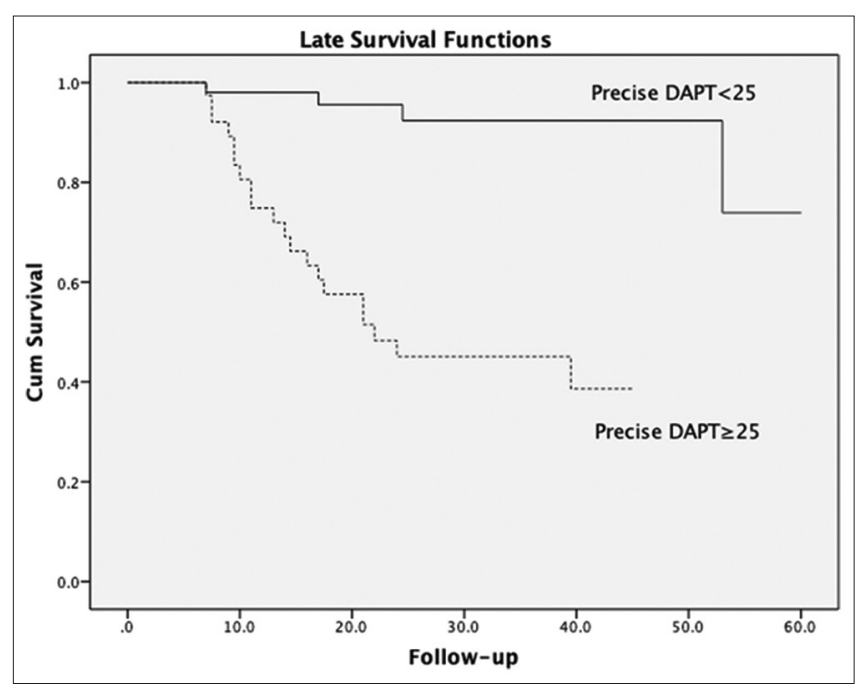

Figure 3: Kaplan Meier late survival curve according to PREdicting bleeding Complications In patients undergoing Stent implantation and subsEquent Dual Anti-Platelet Therapy

The incidence of heart failure increases with age. ${ }^{[11]}$ Moreover, the Framingham study showed that advanced age is a predictor of shortened survival in individuals with congestive heart failure, showing an increase of $27 \%$ in mortality in men and $61 \%$ in women in each following decade. ${ }^{[12]}$ In addition, the retrospective study by Jong et al. on 38702 patients showed that increasing age is associated with poor prognosis in heart failure. ${ }^{[13]}$ Age increases the frequency in patients with heart failure as well as negatively affects the prognosis.

Anemia, affected by many factors commonly accompanying heart failure, such as chronic inflammation, kidney failure, nutritional deficiency, advanced age, drug use, diabetes mellitus, is present in about one-third of patients with heart failure. Varying degrees of anemia have been reported to increase symptoms of heart failure as well as hospitalization and mortality rates. ${ }^{[14-16]}$

Kidney diseases and heart diseases are among the major health problems worldwide and constitute a complex structure. GFR has been defined by the National Kidney Foundation practice guidelines as the best measurement tool for assessing kidney functions and has taken the first place in the evaluation of kidney functions. ${ }^{[17]}$ It has been shown that while the main cause of death in nondialysis-dependent chronic kidney disease is CAD and there is a significant correlation between the level of GFR and the severity of CAD.$^{[18]}$ Epidemiological studies have reported that renal dysfunction secondary to neurohormonal, parenchymal edema-injury, congestion or ischemia, secondary to medication is frequently present in $17 \%-30 \%$ of patients with acute heart failure. Worsening heart failure is associated with worsening kidney function and often exacerbates the other. The correlation of low GFR and high BUN levels with increased mortality and morbidity in patients with heart failure has been demonstrated by many studies. ${ }^{[19-21]}$

The white blood cell count, also called leukocytes, is one of the three main types of blood cells in the blood and is

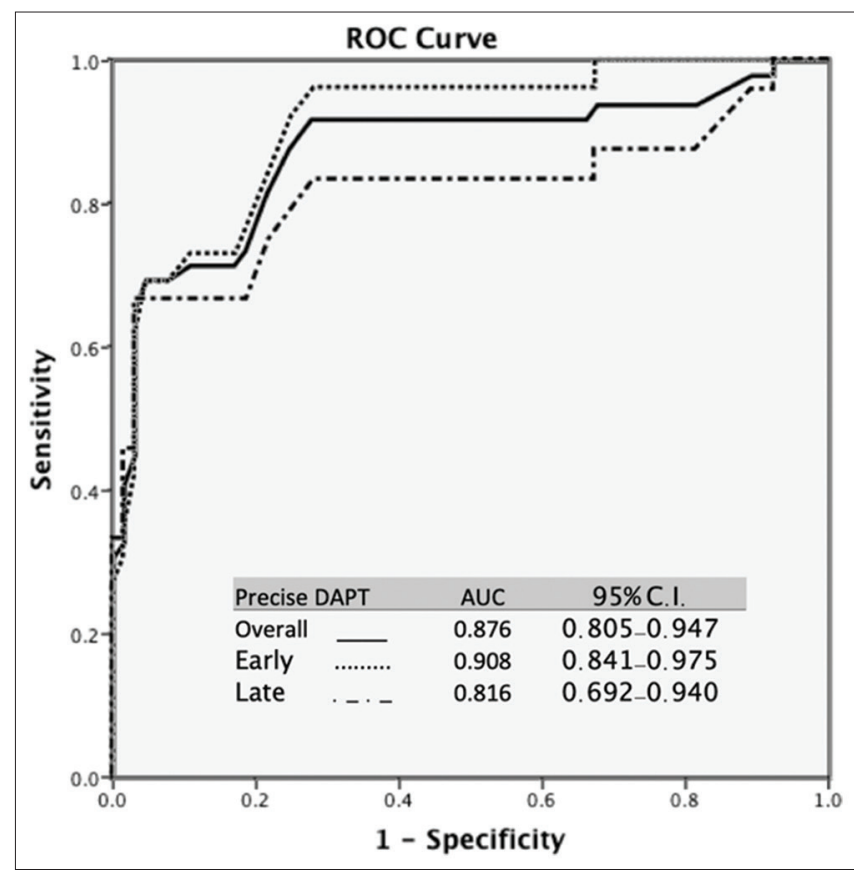

Figure 4: Receiver operating characteristic Curves according to PREdicting bleeding Complications In patients undergoing Stent implantation and subsEquent Dual Anti-Platelet Therapy score cut-off

considered as one of the indicators of inflammation in the body. ${ }^{[22]}$ Chronic inflammation level has been associated with serious health problems such as chronic heart disease, diabetes mellitus, and malignancies, and the large observational study by Menon et al. showed that peripheral total leukocyte count was strongly correlated with heart failure, cardiogenic shock and mortality during hospitalization of patients with acute myocardial infarction (MI). It has been shown to be somewhat related. ${ }^{[23]}$ Acute and chronic inflammation plays an active important pathophysiological role in the onset and progression of heart failure and other cardiovascular diseases. Stimulation of inflammation stimulates proteolytic enzyme and pro-inflammatory cytokines, then elevated cytokines and inflammation levels can cause myocardial depression, cardiac dysrhythmias, and myocardial remodeling, which may result in heart failure. In previous studies, inflammatory cytokines such as C-reactive protein, interleukin (IL)-4 IL-6 have been shown to be associated with increased mortality. ${ }^{[24,25]}$ In addition, inflammation has been shown to play an active role in the pathophysiology of both acute and chronic heart failure, and it has been emphasized that treatment management can focus on treatments for inflammation, and inflammatory markers can be used to predict prognosis. ${ }^{[24-26]}$

An increase in minor or major bleeding is an expected picture in cardiovascular diseases, due to single, double, or triple antiaggregant and anticoagulant therapy, which are frequently used for reasons such as valvular lesions, arrhythmia, CAD, and thrombus compared to patients not receiving this treatment. Especially even isolated major bleeding-related mortality can be high, the history of previous bleeding in patients with 
heart failure can be considered as a poor prognostic factor and indicator for recurrent bleeding.

Recent studies have shown that there may be a close correlation between PRECISE-DAPT and cardiovascular events. The study by Yildirim et al. included 706 ST Segment Elevation MI patients and showed a significant correlation between PRECISE-DAPT and high-degree AV block and AF. ${ }^{[27]}$

In another study by Çınar et al., the correlation between the development of contrast nephropathy was investigated in patients undergoing primary percutaneous intervention with the diagnosis of ST elevation. The study included 1280 patients undergoing percutaneous coronary intervention with the diagnosis of MI and the postoperative 72-h creatinine values were evaluated to calculate contrast nephropathy and categorized as contrast-induced nephropathy (CIN) and non-CIN. As a result, it was found that there was a significant correlation between the risk of developing CIN in the group with high PRECISE-DAPT score. ${ }^{[28]}$

PRECISE-DAPT score is a new scoring system that determines the duration of bilateral antiplatelet therapy for patients undergoing coronary intervention, in which patients are classified by the risk of bleeding. Patients with a PRECISE-DAPT score $\geq 25$ have a higher risk of bleeding, and therefore shorter-term bilateral antiplatelet use is recommended. Heart failure is a multifactorial process, increasing frequency with age, often accompanied by comorbidities such as anemia, kidney failure, a history of bleeding due to the use of multiple antiplatelet or anticoagulant drugs, and inflammation contributes to an important pathophysiological process in both acute and chronic stages. Given all of the above risk factors, the parameters age, hemoglobin level, GFR value, leukocyte count and previous bleeding history that make up the PRECISE-DAPT score are also the factors that contribute to the heart failure prognosis. ${ }^{[6]}$ The predictive value of mortality of PRECISE-DAPT score in patients with ADHF is uncertain, and this study may be the first study to show that PRECISE-DAPT score can be considered as a mortality predictor in patients with ADHF.

In addition, the precise DAPT score was also compared with the GWTG heart failure risk score, a heart failure mortality score, and was not statistically significant, although it was close to the significance value. This correlation may not have been found due to the small number of patients.

PRECISE DAPT is a score that can be calculated easily and does not require an additional medical examination. Heart failure is also a chronic disease that we often encounter in emergency departments and still has a high mortality rate. PRECISE DAPT can be easily calculated in the first application and we can schedule more frequent examinations and increase treatment for mortality based on the risk score. Medical or device treatment may be planned earlier. But to use this score routinely, we need further studies with long-term follow-up and large-scale prospective data.

\section{Study limitations}

Our study has some limitations. First, the most important limitations are retrospective design and small sample size. Second, we were only able to assess the overall cause and mortality due to the lack of data on the causes of death. Third, due to the lack of data, the correlation of PRECISE DAPT with other heart failure mortality scores could not be evaluated. Forth, due to lack of data, BNP or NT-proBNP measurements were not reported.

In order to explain the correlation between mortality and PRECISE-DAPT score in patients with acute decompensated cardiac disease, there is a need for multicenter, prospective, and longer-term studies with a larger sample size.

\section{Conclusion}

As far as we know, our study is the first study evaluating the correlation between PRECISE-DAPT and mortality in patients with acute decompression heart failure. PRECISE-DAPT score is an easy to memorize scoring system that can be easily calculated in emergency departments, even in rapidly developing cardiovascular events, and does not contain any parameter other than routine biochemical evaluations. In acute decompensated patients whose short and long-term mortality is still high, it may predict to differentiate the group of patients with higher mortality during initial medical contact or hospitalization process and to consider more intensive follow-up or further treatment for this population.

\section{Declaration of patient consent}

The authors certify that they have obtained all appropriate patient consent forms. In the form the patients have given their consent for their images and other clinical information to be reported in the journal. The patients understand that their names and initials will not be published and due efforts will be made to conceal their identity, but anonymity cannot be guaranteed.

\section{Financial support and sponsorship}

Nil.

\section{Conflicts of interest}

There are no conflicts of interest.

\section{References}

1. Farahmand $\mathrm{S}, \mathrm{Abdolhoseini} \mathrm{A}$, Aliniagerdroudbari E, Babaniamansour $\mathrm{S}$, Baratloo A, Bagheri-Hariri S. Point-of-care ultrasound modalities in terms of diagnosing acute decompensated heart failure in emergency department; a diagnostic accuracy study. Intern Emerg Med 2020;15:491-9.

2. Ponikowski P, Voors AA, Anker SD, Bueno H, Cleland JG, Coats AJ, et al. 2016 ESC Guidelines for the diagnosis and treatment of acute and chronic heart failure: The Task Force for the diagnosis and treatment of acute and chronic heart failure of the European Society of Cardiology (ESC). Developed with the special contribution of the Heart Failure Association (HFA) of the ESC. Eur J Heart Fail 2016;18:891-975.

3. Joseph SM, Cedars AM, Ewald GA, Geltman EM, Mann DL. Acute decompensated heart failure: Contemporary medical management. Tex Heart Inst J 2009;36:510-20. 
4. Natella PA, Le Corvoisier P, Paillaud E, Renaud B, Mahé I, Bergmann JF, et al. Long-term mortality in older patients discharged after acute decompensated heart failure: A prospective cohort study. BMC Geriatr 2017; $17: 34$.

5. Lloyd-Jones D, Adams RJ, Brown TM, Carnethon M, Dai S, De Simone G, et al. Executive summary: Heart disease and stroke statistics-2010 update: A report from the American Heart Association. Circulation 2010;121:948-54.

6. Choi SY, Kim MH, Cho YR, Sung Park J, Min Lee K, Park TH, et al. Performance of PRECISE-DAPT Score for Predicting Bleeding Complication During Dual Antiplatelet Therapy. Circ Cardiovasc Interv 2018; $11: \mathrm{e} 006837$.

7. McKee PA, Castelli WP, McNamara PM, Kannel WB. The natural history of congestive heart failure: The Framingham study. N Engl J Med 1971;285:1441-6.

8. Tokgözoğlu L, Yılmaz MB, Abacı A, Altay H, Atalar E, Aydoğdu S, et al. Heart Failure Roadmap in Turkey: Recommendations for policies that can be developed to prevent heart failure and related deaths. Turkish Society of Cardiology. 2017. Available from: https://www.tkd.org.tr/ TKDData/Uploads/files/Turkiyede-kalp-yetersizligi-yol-haritasi.pdf. [Erişim: 2017 Apr 24].

9. Karakuş A, Berat UĞ. Prognostic value of the Selvester QRS score for re-hospitalization in patients with ischemic heart failure. J Surg Med 2020;4:1165-8.

10. Heo S, Lennie TA, Okoli C, Moser DK. Quality of life in patients with heart failure: Ask the patients. Heart Lung 2009;38:100-8.

11. Kurmani S, Squire I. Acute heart failure: Definition, classification and epidemiology. Curr Heart Fail Rep 2017;14:385-92.

12. Ho KK, Anderson KM, Kannel WB, Grossman W, Levy D. Survival after the onset of congestive heart failure in Framingham Heart Study subjects. Circulation 1993;88:107-15.

13. Jong P, Vowinckel E, Liu PP, Gong Y, Tu JV. Prognosis and determinants of survival in patients newly hospitalized for heart failure: A population-based study. Arch Intern Med 2002;162:1689-94.

14. Tang WH, Tong W, Jain A, Francis GS, Harris CM, Young JB. Evaluation and long-term prognosis of new-onset, transient, and persistent anemia in ambulatory patients with chronic heart failure. J Am Coll Cardiol 2008;51:569-76.

15. Waldum B, Westheim AS, Sandvik L, Flønæs B, Grundtvig M, Gullestad L, et al. Baseline anemia is not a predictor of all-cause mortality in outpatients with advanced heart failure or severe renal dysfunction. Results from the Norwegian Heart Failure Registry. J Am Coll Cardiol 2012;59:371-8.
16. Grote Beverborg N, van Veldhuisen DJ, van der Meer P. Anemia in Heart Failure: Still Relevant? JACC Heart Fail 2018;6:201-8.

17. Levey AS, Coresh J, Bolton K, Culleton B, Harvey KS, Ikizler TA, et al. K/DOQI clinical practice guidelines for chronic kidney disease: Evaluation, classification, and stratification. Am J Kidney Dis 2002;39 2 Suppl 1:i-ii+.

18. Chen Q, Zhang Y, Ding D, Xia M, Li D, Yang Y, et al. Estimated Glomerular Filtration Rate and Mortality among Patients with Coronary Heart Disease. PLoS One 2016;11:e0161599.

19. Cleland JG, Carubelli V, Castiello T, Yassin A, Pellicori P, Antony R. Renal dysfunction in acute and chronic heart failure: Prevalence, incidence and prognosis. Heart Fail Rev 2012;17:133-49.

20. Damman K, Testani JM. The kidney in heart failure: An update. Eur Heart J 2015;36:1437-44.

21. Fonarow GC, ADHERE Scientific Advisory Committee. The Acute Decompensated Heart Failure National Registry (ADHERE) Opportunities to improve care of patients hospitalized with acute decompensated heart failure. Rev Cardiovasc Med 2003;4 Suppl 7:S21-30.

22. Kounis NG, Soufras GD, Tsigkas G, Hahalis G. White blood cell counts, leukocyte ratios, and eosinophils as inflammatory markers in patients with coronary artery disease. Clin Appl Thromb Hemost 2015;21:139-43

23. Menon V, Lessard D, Yarzebski J, Furman MI, Gore JM, Goldberg RJ. Leukocytosis and adverse hospital outcomes after acute myocardial infarction. Am J Cardiol 2003;92:368-72.

24. Durmus E, Kivrak T, Gerin F, Sunbul M, Sari I, Erdogan O. Neutrophil-to-lymphocyte ratio and platelet-to-lymphocyte ratio are predictors of heart failure. Arq Bras Cardiol 2015;105:606-13.

25. Prabhu SD. Cytokine-induced modulation of cardiac function. Circ Res 2004;95:1140-53.

26. Shirazi LF, Bissett J, Romeo F, Mehta JL. Role of Inflammation in Heart Failure. Curr Atheroscler Rep 2017;19:27

27. Yildirim E, Turkkan C, Ozcan KS, Ceylan US, Zengin A. The predictive value of PRECISE-DAPT score for arrhythmic complications in patients with ST-elevation myocardial infarction. Coron Artery Dis 2019;30:499-504.

28. Çınar T, Tanık VO, Aruğaslan E, Karabağ Y, Çağdaş M, Rencüzoğulları İ, et al. The association of PRECISE-DAPT score with development of contrast-induced nephropathy in patients with ST-elevation myocardial infarction undergoing primary percutaneous coronary intervention. Cardiovasc Interv Ther 2019;34:207-15. 